\title{
Simulation versus Design: Two Case Studies in Chemical Reaction Kinetics and Radiative Heat Transfer
}

\author{
Redhouane Henda \\ School of Engineering, Laurentian University, Sudbury, ON P3E 2C6, Canada \\ Email: rhenda@laurentian.ca
}

\begin{abstract}
This paper describes two instructional modules pertaining to engineering design education and developed via two design approaches. The first module concerns itself with experimental simulation of chemical reaction kinetics using the analogy with the free-fall of a non-viscous liquid in ducts of various shapes. The second module is concerned with computer simulation of heat exchange via thermal radiation in a 3-D enclosure with nontrivial features. Both modules involve a series of case studies to enhance students understanding of the engineering concepts under consideration and to expose them to the power of both experimental and computer designs.
\end{abstract}

\section{Introduction}

Laboratory work reinforces the theoretical foundation of courses laid out during formal lectures, and it is a way to help students develop vital experimental skills such as planning, observation, analysis, communication, etc., to succeed in their future careers [1-3]. This strategy of learning skills through "doing", however, is not always an easy task because of the limitations often imposed by high costs, incurred from equipment maintenance, and safety requirements. These shortcomings can be countered by resorting to simulation and experimental design tools. In other words, expensive and/or hazardous experiments can be simulated by simple physical concepts whose mathematical models are similar in form to the original physical concept one is seeking to investigate. This idea has been exploited in this study to simulate the kinetics of simple chemical reactions via experimental design and investigation of the hydrodynamics of the draining of ducts of various geometric shapes.

Of equal importance in engineering design education is the development of computer modules to supplement classroom presentations [4]. A key factor is the identification of curriculum activities that cannot be easily accomplished by experimental means and/or solved analytically. Models of sufficient fidelity and execution time provide students with opportunities in reflection on, evaluation and justification of decisions in engineering design [5, 6]. For the purpose of this study the problem of thermal radiation exchange in a 3-D enclosure is considered. This choice has been dictated by many factors: First, by the lack of any analogy with the other modes of transport (mass, heat, and momentum), which is not helpful from the pedagogic viewpoint, and, second, by the strong nonlinearity of the equations describing thermal radiation, making their solution by analytical means impossible and numerically quite difficult, especially for realistic geometries and surfaces.

This paper describes two modules in chemical reaction kinetics (module I) and thermal radiation (module II), and developed using a combination of concepts based on the principles of simulation and design. The next sections give descriptions of the modules along with the discussion of some related case studies. Both modules can serve as useful educational tools in courses in chemical reaction kinetics and heat transfer, and provide excellent exercises for undergraduate students to reflect on, evaluate and justify decisions in engineering design.

\section{Modules description}

Module I: Chemical Reaction Kinetics

Model: The objective of the experimental module is to study the kinetics of simple chemical reactions in a closed well-stirred tank reactor by analogical hydrodynamic models. A simple chemical reaction of the form $\mathrm{A} \rightarrow$ products has a reaction rate, $\mathfrak{R}$, which can be expressed as [7]

$$
\Re=-\frac{d C_{A}}{d t}=k C_{A}^{n},
$$

where $k$ and $n$ are the kinetic constants, $C_{A}$ is the concentration of species $\mathrm{A}$, and $t$ is time. 


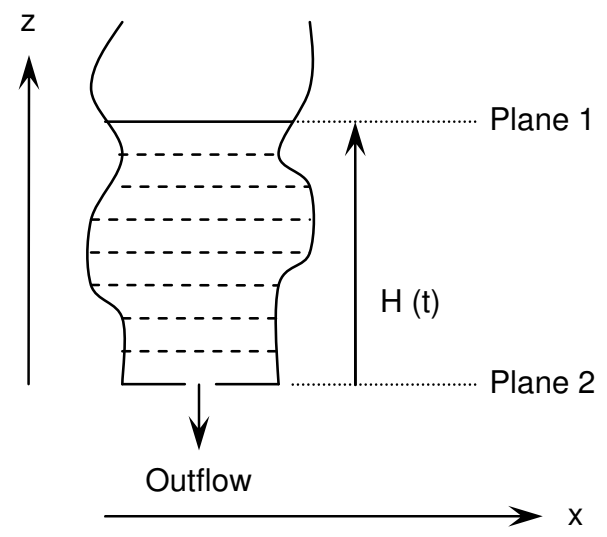

Figure 1: Physical system

Consider the isothermal system (duct) illustrated in Figure 1 through which a non-viscous liquid flows and drains through a small orifice. The hydrostatic height $H$ of the liquid decreases with time as the volume of the liquid in the system decreases and is not replaced. The flow is clearly unsteady. From the macroscopic expressions of the energy and mass balances within the boundaries of the system, between plane 1 and plane 2 , it is easy to establish the relationship between the velocity of the liquid, $v_{2}$, at the outlet of the cylinder, the velocity, $v_{l}$, of the falling liquid at the liquid free surface, and the hydrostatic height, $H$. Assuming frictional losses to be negligible and the liquid density, $\rho$, to be constant, the balances are as follows.

Energy balance (Bernoulli equation):

$$
\Delta \frac{v^{2}}{2}+g \Delta H=0
$$

Mass balance:

$$
-\frac{d m}{d t}=-\rho A_{1} \frac{d H}{d t}=\rho v_{2} A_{2}=\rho v_{1} A_{1},
$$

In Eq. (2) and Eq. (3) the parameters $A_{1}, A_{2}, g, m$, and $t$ are the cross-sectional area of the duct, outlet orifice area, constant of gravitation, mass of the falling liquid, and time, respectively. If the outlet orifice area, $A_{2}$, is much smaller than the area of the liquid free surface, $A_{l}$, the velocity of the liquid in the duct, $v_{l}$, is small compared with the velocity of the liquid leaving the duct, $v_{2}$. Under this condition the term $v_{l}^{2}$ can be neglected in Eq. (2). This assumption holds as long as the liquid free surface is far from the outlet, namely for large enough values of $z$. A substitution of $v_{2}$ obtained from Eq. (3) into Eq. (2) leads to the differential form of the Bernoulli equation, Eq. (2), as

$$
-\frac{d H}{d t}=\sqrt{2 g} \frac{A_{2}}{A_{1}} H^{1 / 2}
$$

If the duct has a constant depth, $\delta$, and the height, $H$, is assumed to be related to the width, $x$, according to the equation

$$
H=\alpha x^{\beta},
$$

where $\alpha$ and $\beta$ are constants, the cross-sectional area, $A_{l}$, can then be expressed as

$$
A_{1}=\delta x=\delta\left(\frac{H}{\alpha}\right)^{1 / \beta}
$$

Substituting Eq. (6) in Eq. (4) leads to the final differential form of the Bernoulli equation,

$$
-\frac{d H}{d t}=\sqrt{2 g} \frac{A_{2} \alpha^{1 / 2}}{\delta} H^{(1 / 2-1 / \beta)}
$$

The analogy between the kinetics of simple chemical reactions and the hydrodynamics of duct drainage is now established - compare Eq. (1) to Eq. (7) whereby the equivalent variables are $C_{A}$ and $H$ - an adequate choice of the value of $\beta$ yields a series of case studies. For instance, a reaction of order " 0 " can be simulated by a duct whose lateral boundary is a parabola, a reaction of order " $1 / 2$ " can be simulated with a duct whose lateral boundary is a vertical line ( $\beta$ $>>1$ ), e.g., a cylinder is a good example, and a reaction of order "-1/2" can be simulated with a duct whose lateral boundary is linear in form.

Experimental Procedure: Students are asked to establish the analogy discussed in the previous section, to choose the order of the simulated chemical reaction and to design the corresponding duct (Plexiglas material is used for that purpose) according to the assumptions made in model development. Thereafter, they will carry out a set of experimental as per the following procedure.

1. Set up the ducts on a vertical panel along with the necessary accessories, e.g., beakers, scales, dyes, stop watch, etc.

2. Fill the duct with a non-viscous liquid, e.g., water, until the zero reading of the scale.

3. Make sure air bubbles in the duct are eliminated, and refill the duct with the liquid until the zero reading.

4. Open the orifice and start recording the distance travelled by the liquid free surface, $D T_{i}$ as a function of time $t_{i}$ ( $i$ refers to the number of reading). Either a 
Table 1: Data generation

\begin{tabular}{|l|l|l|l|}
\hline Run i & $\begin{array}{l}\text { Distance traveled } \\
\text { by the liquid free- } \\
\text { surface, } \mathrm{DT}_{\mathrm{i}}(\mathrm{mm})\end{array}$ & $\begin{array}{l}\text { Time, } \\
\mathrm{t}_{\mathrm{i}}(\mathrm{s})\end{array}$ & $\begin{array}{l}\text { Actual } \\
\text { height, } \mathrm{H}_{\mathrm{i}} \\
(\mathrm{mm})\end{array}$ \\
\hline 1 & & & \\
\hline 2 & & & \\
\hline 3 & & & \\
\hline $\mathrm{m}-1$ & & & \\
\hline $\mathrm{m}$ & & & \\
\hline
\end{tabular}

time or a liquid height increment can be fixed during the experiment.

5. Take frequent readings and tabulate the results as time vs. height as shown in Table 1 , where $H_{i}=$ height of the duct $-D T_{i}$.

6. Repeat steps 1 through 5 for a number of times for a statistical analysis of the results.

7. Find graphically the order $\eta$ and constant $\chi$ for each of the models considered.

8. Use a software package (e.g., Matlab [8], Maple [9], etc.) to determine the order $\eta$ and constant $\chi$ using the Simplex optimization technique [10] (for instance, in case of Matlab, it is available through the library function fminsearch). The function objective to be entered in the software package is based on the principle of least squares and defined by

$$
\mathrm{F}(\eta, \chi)=\sum_{i=m}\left[\left(-\frac{d H}{d t}\right)_{i}-\chi H_{i}^{\eta}\right]^{2},
$$

with

$$
\eta=1 / 2-1 / \beta \quad \text { and } \quad \chi=\sqrt{2 g} \frac{A_{2} \alpha^{1 / 2}}{\delta},
$$

where $m$ is the total number of readings.

9. Compare graphical and software package results to theoretical predictions.

10. Submit a report on your findings.

\section{Module II: Radiative Heat Transfer}

Model: The objective of the computer module is to simulate heat transfer by radiation in a $3-D$ rectangular enclosure (dimensions: XxYxZ) using a Monte Carlo technique. Both concepts of view and exchange factors, the backbones of any thermal radiation analysis [11], are considered. The enclosure contains a substrate (dimensions: XsxYsxZs) and a filament as sketched in Figure 2.

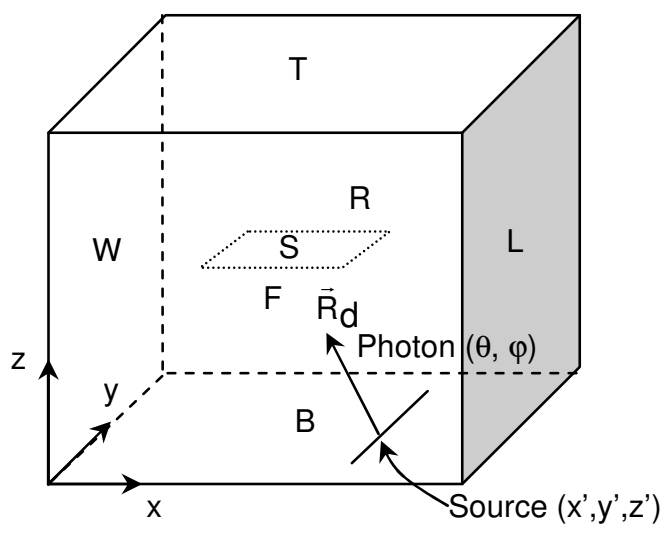

Figure 2: Physical system

One of the more efficient approaches for thermal radiation analysis is the Monte Carlo (MC) method because of its ability to tackle complex problems with relative ease compared to other numerical techniques [12-19]. Generally, undergraduate students are not exposed to stochastical techniques, although their implementation is quite intuitive and does not require beyond the physics of the problem at hand along with a standard background in statistics. Analyzing a thermal radiation problem using a MC method consists of tracing the history of a meaningful sample of energy bundles (photons) from their emission to their eventual absorption. Sampling of the source of energy, direction and wavelength of bundle, absorption or reflection of bundle, etc., is accomplished using probability distributions. For instance, the sampling of energy bundles of the same wavelength, $\lambda$, is done by randomly selecting a fraction of the total emitted energy, $E_{\lambda}$, over all wavelengths between 0 and $\infty$ (see Figure 3 ) defined by

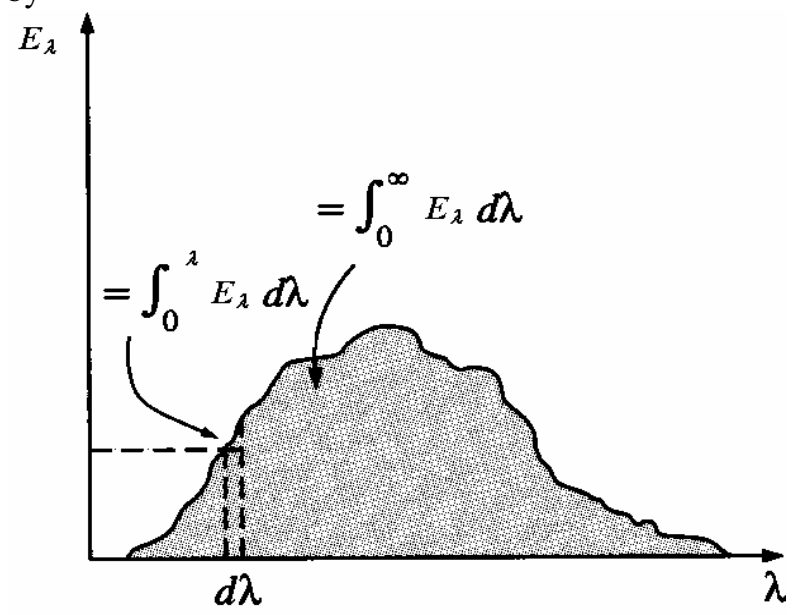

Figure 3: Probability distribution for $E_{\lambda}$. 


$$
\mathrm{R}(\lambda)=\int_{0}^{\lambda} P(\lambda) d \lambda=\frac{\int_{0}^{\lambda} E \lambda d \lambda}{\int_{0}^{\infty} E \lambda d \lambda},
$$

where the function $R$ defines a random number in $\mathrm{MC}$ calculations.

Inverting Eq. (8) yields the wavelength of the bundle, e.g., according to the polynomial expansion given by Haji-Sheikh [20]. Similarly, the position of emitting source, direction of bundle, and its absorption/reflection behaviour are sampled from equivalent probability distributions as follows.

Source, S:

$$
\xi_{S}=R_{S}\left(\xi_{2}-\xi_{1}\right)+\xi_{1},
$$

where $\xi_{s}=\left(x^{\prime}, y^{\prime}, z^{\prime}\right), 1$ and 2 correspond to the endpoints defining the filament segment, and $R s$ is a random number.

Direction of bundle:

$$
\begin{aligned}
& \theta_{S}=\cos ^{-1}\left(1-2 R_{\theta}\right), \\
& \theta_{\text {Surf }}=\sin ^{-1}\left(\sqrt{R_{\theta}}\right), \\
& \varphi_{S, \text { surf }}=2 \pi R^{R} \varphi
\end{aligned}
$$

where $R_{\theta}$ and $R_{\varphi}$ are random numbers.

Position of bundle: It is defined in terms of its origin and the direction vector $R_{d}$ by

$$
\xi=\xi_{S}+t R_{d}
$$

where $\xi=(x, y, z)$ and $t$ is a positive number.

For an energy bundle to hit one of the planar surfaces making up the enclosure the following equation must be fulfilled

$$
A x+B y+C z+D=0
$$

where $A, B, C$ and $D$ are constants defining the planar surface in question.

Absorption/reflection of bundle: The condition of absorption of an energy bundle by a surface is given by

$$
R_{\alpha} \leq \alpha \lambda
$$

where $\alpha_{\lambda}$ is the surface absorptivity at wavelength $\lambda$. In this case a new source is randomly selected on the filament and the bundle is traced as per Eqs. (8)-(13). Otherwise the bundle is reflected from the surface and is further traced according to Eqs. (10)-(13).

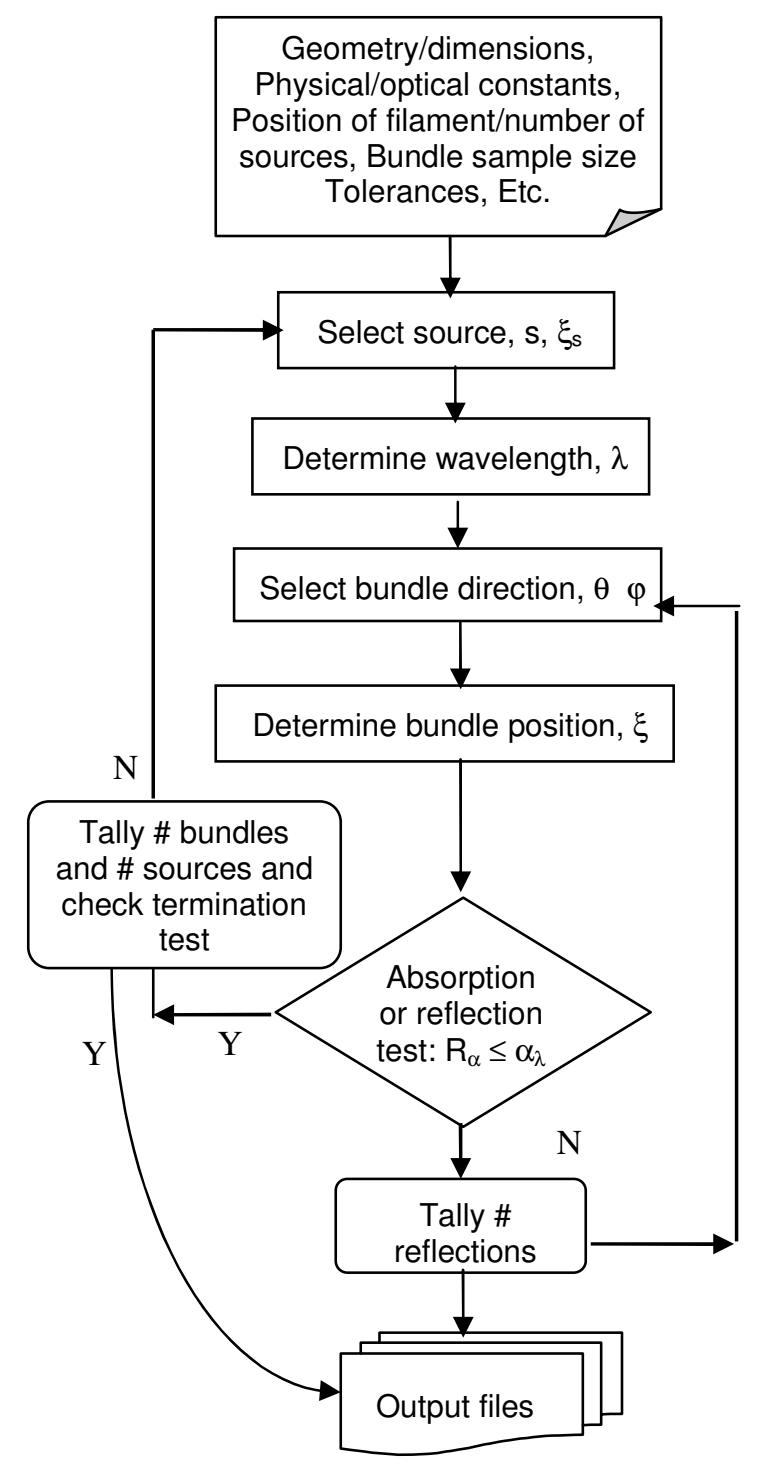

Figure 4: Simplified module flowchart

The view (black body case) or exchange factor $F_{s-i}$ between a source $s$ and a surface $i$ is defined as

$$
F_{S-i}=\frac{\text { Numberof bundlesabsorbedby surface } i}{\text { Total numberof emittedbundlesby sources }}
$$

The total view or exchange factor between a filament and a surface $i$ is calculated by integrating Eq. (14) over a large number of energy bundles emitted by the filament.

Numerical Procedure: After data on enclosure geometry and physical properties have been defined, a series of computer experiments can be carried out according to the flowchart shown in Figure 4. Students are asked to solve a series of case studies 
with the goal of exposing them to various thermal radiation concepts corresponding to black, diffusegray and diffuse-nongray surface approximations. In the end the students should be able to assess the importance of the various design parameters on the performance of the enclosure.

\section{Results and discussion}

Module I: Various simple chemical reactions can be simulated by means of the hydrodynamic analog along with the design approach described early in the previous section. Plexiglas and other rudimentary laboratory materials are used to design the hydrodynamic models. After the models have been designed, the suggested experimental procedure is undertaken to generate data relative to different simulated kinetic models as desired.

A typical example is shown in Figure 5 for the case of the hydrodynamics with parameter $\beta=1$ and corresponding to a simple chemical reaction of order $\eta$ $=-1 / 2$. Figure 5 depicts the experimentally measured hydrostatic height of the free-falling liquid as a function of draining time. In order to determine the design parameters $\eta$ and $\chi$ graphically the liquid velocity, $-d H / d t$, is plotted as a function of its height, $H$, as illustrated in Figure 6. A power function fit of the experimental data yields values of $\sim 13.08$ and $\sim$ 0.53 (theoretical value $=0.5$ ) for the parameters $\chi$ and $\eta$, respectively. These same parameters have also been estimated using the efficient Simplex optimization technique [10] available in Matlab. The calculated values are $\sim 14.44$ and $\sim-0.55$ for the parameters $\chi$ and $\eta$, respectively (see appendix for further details). The values of the parameters

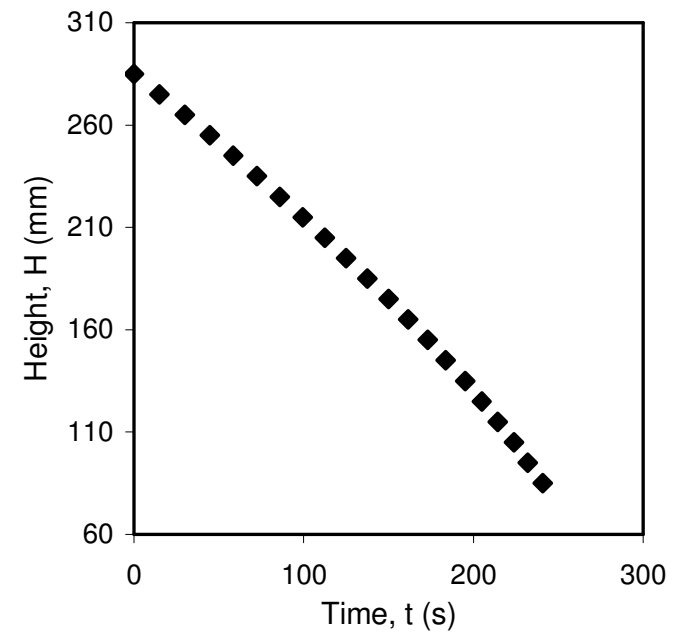

Figure 5: Liquid hydrostatic height vs. draining time for a model of order $-\mathbf{1 / 2}$.

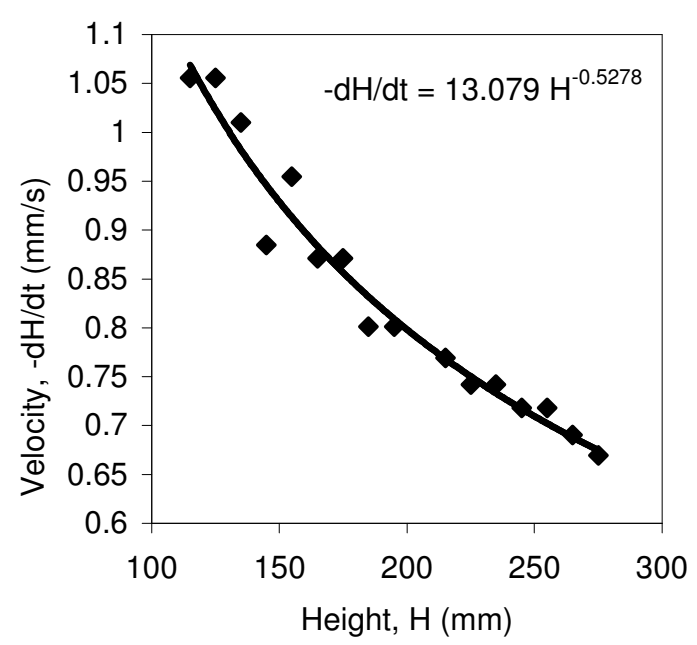

Figure 6: Liquid velocity vs. hydrostatic height for a model of order $-1 / 2$. The solid line is a power fit of experimental data.

estimated by means of graphical representation and Matlab are in very good agreement with an absolute error of $\sim 10 \%$ for $\chi$ and of $\sim 4 \%$ for $\eta$.

Module II: In the present study the following configuration parameters along with pertinent physical properties have been used: enclosure dimensions $X / Z$ $=Y / Z=1$, substrate dimensions $\mathrm{Xs} / \mathrm{Z}=\mathrm{Ys} / \mathrm{Z}=\mathrm{Zs} / \mathrm{Z}=$ 0.5, filament temperature $T=2700 \mathrm{~K}$, surface absorptivity $\alpha=1$ (black body), $\alpha=0.45$ (diffusegray) and $\alpha_{\lambda}=0.45$ for $\lambda \leq 1.0 \mu \mathrm{m}$ and $\alpha_{\lambda}=0.15$ elsewhere (diffuse-nongray), filament position $\left(x_{1} / X=\right.$ $\left.0.5, y_{1} / Y=0.25, z_{1} / Z=0.1\right)$ and $\left(x_{2} / X=0.5, \quad y_{2} / Y=\right.$ $\left.0.75, z_{2} / Z=0.1\right)$. Any departure from these conditions is pointed to when appropriate. Only typical examples are given hereafter.

First, the effect of sampling on the accuracy of MC calculations has been assessed. To this end, the size of energy bundle sample has been increased between the values of 1000 and 10000 and recorded against the corresponding calculated values of the view factor. For simplicity a single source located at the center of the enclosure (substrate, $S$, is omitted) and the black body approximation are considered here. The results of the calculations are shown in Table 3 for all enclosure surfaces. As one can notice from Table 3 both stability and accuracy of MC calculations improve with increasing bundle sample size and reach the analytical solution for this case for a sample size of 100,000 (this is the size used for all further calculations). Since the source is located at the center of the enclosure then the analytical solution, which is quite intuitive, yields a value of $1 / 6$ for all view factors 
Table 3: Effect of bundle size on calculation accuracy of view factors for all enclosure walls.

\begin{tabular}{r|rrrrrr|}
\cline { 2 - 7 } & \multicolumn{1}{c}{$\mathrm{T}$} & $\mathrm{L}$ & $\mathrm{B}$ & $\mathrm{W}$ & $\mathrm{R}$ & \multicolumn{1}{c}{$\mathrm{F}$} \\
\cline { 2 - 7 } 1000 & 0.171 & 0.174 & 0.167 & 0.177 & 0.16 & 0.151 \\
10000 & 0.1701 & 0.1589 & 0.1732 & 0.1677 & 0.1632 & 0.1669 \\
20000 & 0.1682 & 0.1634 & 0.1708 & 0.1702 & 0.1607 & 0.1668 \\
30000 & 0.17 & 0.16217 & 0.16977 & 0.1688 & 0.1631 & 0.1662 \\
50000 & 0.1677 & 0.16494 & 0.16834 & 0.1674 & 0.1643 & 0.1673 \\
80000 & 0.1673 & 0.16538 & 0.16664 & 0.1673 & 0.1664 & 0.167 \\
90000 & 0.1662 & 0.16597 & 0.16754 & 0.1667 & 0.1661 & 0.1674 \\
1 E+05 & 0.1662 & 0.16643 & 0.16666 & 0.167 & 0.1667 & 0.1669 \\
Exact & 0.1667 & 0.16666 & 0.16666 & 0.1667 & 0.1667 & 0.1667 \\
\cline { 2 - 6 } & & & & & &
\end{tabular}

between the source and the surrounding surfaces. The distribution of energy bundles absorbed by surface $T$ is shown in Figure 7. As expected the distribution is symmetrical with the maxim amount of energy intercepted by the central region of the surface, which is closest to the source. In the following, the substrate $S$ is included in the enclosure and placed at the center of the enclosure. Figure 8 depicts the number of bundles emitted by the filament and absorbed by $S$ for the diffuse-gray and diffuse-nongray conditions given in the previous paragraph. The effect of the heating filament on energy bundle distribution can be clearly seen (oval-shaped contours in Figure 8). The effect of surface absorptivity on energy bundle distribution can also be assessed.

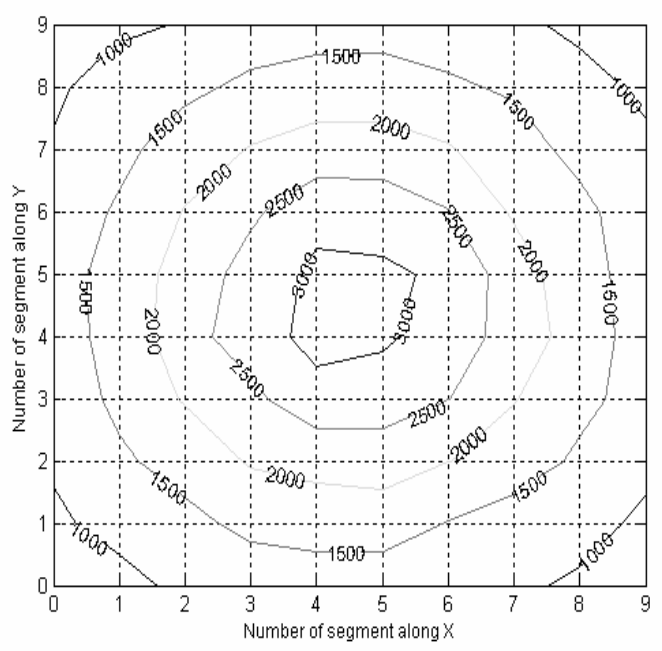

Figure 7: Distribution of energy bundles absorbed by surface $T$ for a black body enclosure with a central source.
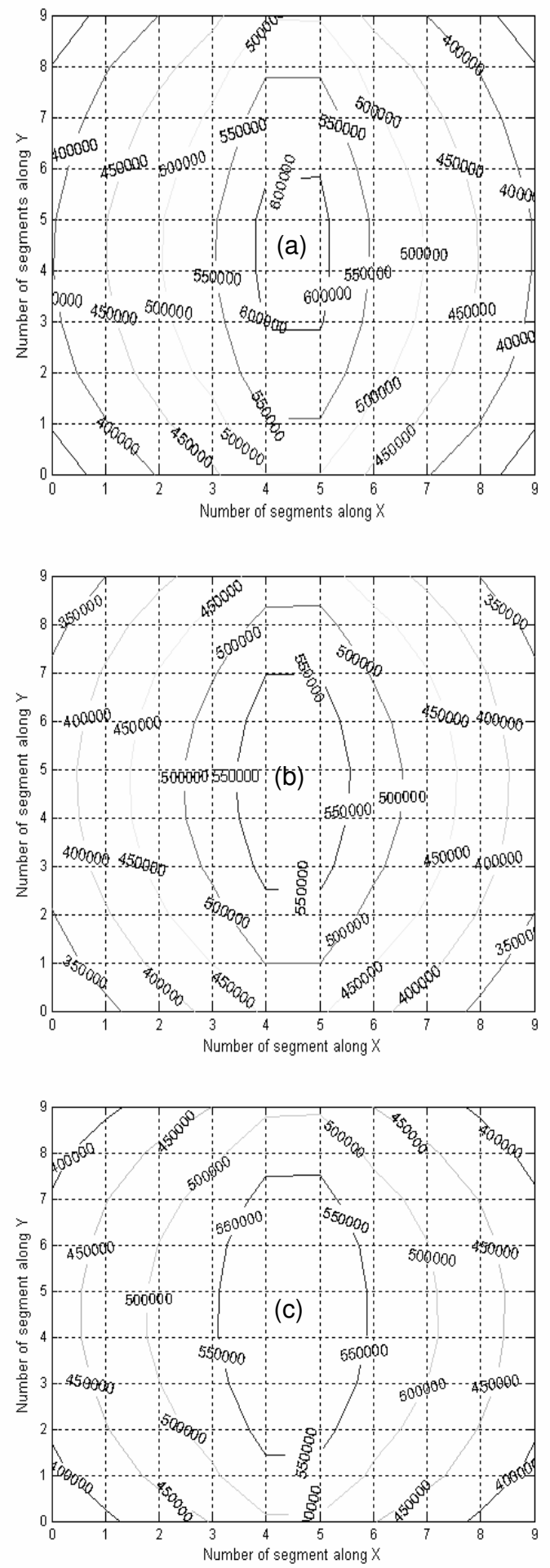

Figure 8: Distribution of energy bundles absorbed by surface $S$ for the diffuse-gray case (a) $\alpha=0.45$ and (b) $\alpha=0.15$, and diffusenongray case (c) $\alpha_{\lambda}$ (given in the text). 


\section{Conclusions}

In this paper I report on two modules involving the concepts of simulation and engineering design. The first module simulates the kinetics of simple chemical reactions by means of hydrodynamic analogs. The second module simulates thermal heat exchange in a 3-D enclosure with nontrivial features. Both modules are flexible and allow for the investigation of a variety of design parameters of the applications at hand. They also provide a series of excellent exercises to students by introducing them to different concepts and inviting them to design, analyse and make decisions about the different scenarios.

\section{Acknowledgments}

The author acknowledges the support received from the Natural Science and Engineering Research Council (NSERC) and LURF.

\section{References}

[1] J.G. Mackenzie, R.M. Allen, W.B. Earl, and I.A. Gilmour, "Teaching Creative Problem-Solving Skills in Engineering Design", Chem. Eng. Ed., vol. 33, ASSE, Spring 1999, 151-157.

[2] E.J. Doskocil, "Incorporation of Experimental Design in the Unit Operations Laboratory", Chem. Eng. Ed., vol. 37, ASSE, Summer 2003, 196-201.

[3] B.S. Bloom (Ed.), Taxonomy of Education Objectives: The Classification of Educational Goals. Handbook I: Cognitive Domain, David McKay, Co., New York, NY, 1956.

[4] M.F. Malone, J.A. Trainham, and B. Carnahan (Eds.), Foundations of Computer-Aided Process Design, AIChE Symposium Series, vol. 96, Breckenridge, CO, 2000.

[5] S.R. White, and G. M. Bodner, "Evaluation of Computer-Simulation Experiments in a Senior-Level Capstone Chemical Engineering Course", Chem. Eng. Ed., vol. 33, ASSE, Winter 1999, 34-39.

[6] B. Carnahan (Ed.), Computers in Chemical Engineering Education, CACHE, Austin, TX, 1996.

[7] O. Levenspiel, Chemical Reaction Engineering, $3^{\text {rd }}$ ed., John Wiley, New York, NY, 1999.

[8] Matlab is a trademark of Mathworks, Inc., Natick, MA, USA.

[9] Maple is a trademark of Maplesoft, Waterloo, ON, Canada.

[10] T. Öberg, and S. Deming, "Find Optimum Operating Conditions Fast", Chemical Engineering Progress, vol. 96, AIChE, 2000, 53-59.

[11] J.R. Howell, A Catalog of Radiation Configuration Factors, McGraw-Hill, New York, NY, 1982.

[12] T.J. Chung, and J.Y. Kim, "Radiation View Factors by Finite Elements", J. Heat Transfer, vol. 104, ASME, 1982, 792-795.
[13] A.B. Shapiro, "Computer Implementation, Accuracy and Timing of View Factor Algorithms", J. Heat Transfer, vol. 107, ASME, 1985, 730-732.

[14] A. Ambirajan, and S.P. Venkanteshan, "Accurate Determination of Diffuse View Factors Between Planar Surfaces”, Int. J. Heat Mass Transfer, vol. 36, 1993, 2203-2208.

[15] L.W. Byrd, "View Factor Algebra for Two Arbitrary Sized Non-Opposing Parallel Rectangles", J. Heat Transfer, vol. 115, ASME, 1993, 517-518.

[16] V. Rammohan, and V.M.K. Sastri, "Efficient Evaluation of Diffuse View Factors for Radiation", Int. J. Heat Mass Transfer, vol. 39, 1996, 1281-1286.

[17] S-H Hong, and J.R. Welty, "Monte Carlo Simulation of Radiation Heat Transfer in a Three-Dimensional Enclosure Containing a Circular Cylinder", Num. Heat Transfer, vol. A36, 1999, 395-409.

[18] R. Henda, "Computer Evaluation of Exchange Factors in Thermal Radiation", Chem. Eng. Ed., vol. 38, ASSE, Spring 2004, 126-131.

[19] M..F. Modest, Radiative Heat Transfer, Chapter 19, McGraw-Hill, New York, NY, 1993.

[20] A. Haji-Sheikh, Handbook of Numerical Heat Transfer, W.J. Minkowycz, E.M. Sparrow, G.E. Schneider, and R.H. Plechter (Eds.), John Wiley, New York, NY, 1988.

\section{Appendix}

A patch of Matlab script used to compute $\eta$ and $\kappa$ is given below.

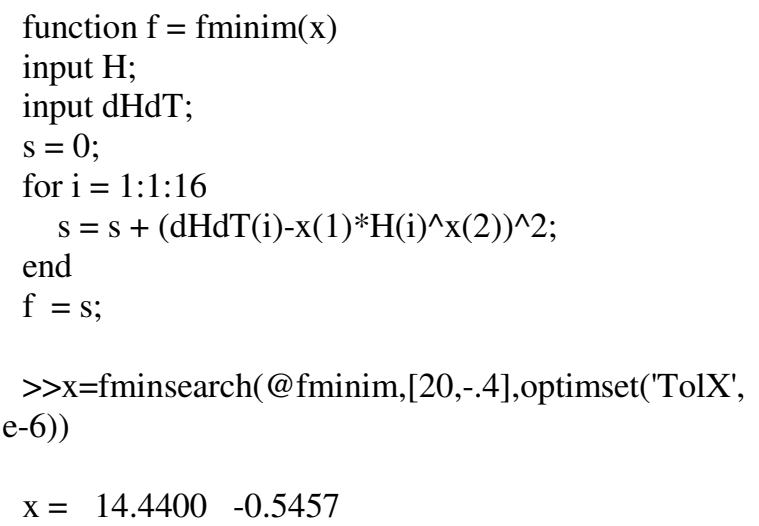

Where the last values correspond to $\eta$ and $\chi$, respectively. 